\title{
REGULACIÓN DE LA INVESTIGACIÓN CLÍNICA Y COMITÉS DE ÉTICA EN PERÚ: CRÓNICA DE CAMBIOS
}

\author{
Agueda Muñoz del Carpio Toia*
}

Resumen: Debido al avance progresivo de la investigación clínica, nuevas exigencias y requerimientos se hacen necesarios, tales como: apertura de comités de ética, creación o adaptación de legislaciones, mayor entrenamiento de investigadores, entre otros.

El objetivo del presente artículo es hacer una crónica de lo sucedido con Reglamento Peruano de Ensayos Clínicos y analizar si la regulación peruana incluye una efectiva participación de la comunidad en los comités de ética.

Palabras clave: bioética, regulación, investigación clínica en Latinoamérica, comités de ética, miembros de la comunidad

\section{REGULATION OF CLINICAL INVESTIGATION IN PERU: CHRONICLE OF CHANGES}

Abstract: Due to the progressive advance of clinical investigation, new demands and requirements are made necessary, such as: the formation of ethics committees, the creation or adaptation of legislation, and increased training for researchers, among others.

The objective of this article is to report on what occurred with the Peruvian Regulation of Clinical Tests and to analyze if the Peruvian regulation includes an effective participation of the community in ethics committees.

Key words: bioethics, regulation, clinical investigation in Latin America, ethics committees, community members

\section{REGULAÇÁO DA PESQUISA CLÍNICA E COMITÊS DE ÉTICA NO PERÚ: CRÔNICA DE MUDANÇAS}

Resumo: Devido ao avanço progressivo da pesquisa clínica, novas exigências e requerimentos se fazem necessários, tais como: criação de comitês de ética, criação ou adaptação de legislaçóes, maior treinamento de pesquisadores, entre outros.

O objetivo do presente artigo é fazer uma crônica do sucedido com o Regulamento Peruano de Ensaios Clínicos e analisar se a regulação peruana inclui uma efetiva participação da comunidade nos comitês de ética.

Palavras-chave: bioética, regulação, pesquisa clínica na América Latina, comitês de ética, membros da comunidade

\footnotetext{
Médico. Profesora e investigadora CICA, Universidad Católica de Santa María, Arequipa Perú.
}

Correspondencia: aguedamunoz@gmail.com. 


\section{Introducción}

Los avances en medicina, biotecnología, tecnología médica, farmacología, entre otros, han permitido disminuir y aliviar el sufrimiento humano causado por múltiples enfermedades, poniendo al alcance de la comunidad nuevas técnicas de abordaje, pruebas diagnósticas, alternativas de tratamiento y hasta posibilidades de terapia basadas en la medicina natural. Todo ello, sin embargo, debe estar normado por principios éticos que protejan el bienestar y los derechos de las personas que participan en la investigación. Los comités de ética de la investigación son los principales responsables de hacer cumplir esta exigencia.

Por muchos años, la ciencia, la investigación y la tecnología intentaron desarrollarse en escenarios sin control ético. La toma de decisiones, la planificación de políticas y la creación e implementación de reglamentaciones no eran el resultado de procesos democráticos. Se debe tener presente que en la investigación médica en seres humanos la preocupación por el bienestar de éstos siempre debe tener primacía por sobre los intereses de la ciencia y de la sociedad.

La comunidad internacional ha entendido el importante rol que juega la ética de la investigación y los comités de ética en la protección de los sujetos de estudio, además de la participación de los representantes de las comunidades en la evaluación de los riesgos y beneficios de una investigación que compromete a seres humanos $(1,2)$.

Perú no es ajeno al desarrollo de la investigación clínica. Actualmente se registra en el país un incremento de los ensayos clínicos. Hasta mayo de 2008 se presentaron en el ente regulador más de 891 ensayos, de los cuales han sido aprobados 800(3). Se le considera el quinto país de Latinoamérica con más protocolos de investigación clínica y la gran mayoría corresponden a patrocinadores de la industria farmacéutica internacional.

Este progreso en las estadísticas se debe a varias razones:

1. Algunas drogas en experimentación podrían ser mejores que los tratamientos usualmente disponibles en el país. No todos los peruanos tienen acceso a un buen seguro de salud y algunas drogas son prohibitivas para la situación económica de muchos pacientes.
2. Es un país que cuenta con centros con altas tasas de enrolamiento de pacientes.

3. Existe acceso a diversos grupos poblacionales sin tratamientos previos.

4. Persisten enfermedades que han sido erradicadas en países desarrollados.

5. Los plazos de los comités y del ente regulador son menores que en otras regiones vecinas y pueden tomar pocas semanas.

6. Se han implementado nuevos comités de ética y diversas oficinas relacionadas con la investigación: CRO (Contract Research Organization) latinoamericanas, entre otras.

Sin embargo, existen grandes problemas en la investigación clínica en Perú. Principalmente, no existen espacios permanentes de capacitación especializada en temas de investigación clínica, regulación de la investigación, buenas prácticas clínicas, auditoría, bioética de la investigación, comités de ética, deberes y derechos en la investigación, entre otros. Todos estos problemas se explican porque no se inicia aún la acreditación de los comités y la certificación de los involucrados en la investigación.

Por otro lado, pese al avance progresivo de la investigación clínica en Perú, recién desde 2006 se reglamentó la investigación clínica en seres humanos, con la aprobación del primer Reglamento de Ensayos Clínicos. En el país, el Instituto Nacional de Salud (INS) es la autoridad encargada de autorizar los ensayos clínicos y de velar por el cumplimiento de las normas que rigen la ejecución de estas investigaciones. En julio de 2006 se aprobó este Reglamento y el 08 de junio de 2007 se aprobó su modificatoria, versión actualmente vigente en Perú $(4,5)$.

La aprobación del Reglamento no contó con la participación de algunos actores relacionados con la investigación y contenía algunos artículos que no eran viables, otros de difícil aplicación y algunos que postergaban los reales derechos de grupos de nuestra sociedad. Por todo ello, el Ministerio de Salud tomó la decisión de llevarlo a consulta pública bajo una propuesta democrática. En el presente artículo se realiza una crónica de lo ocurrido en este proceso.

\section{Objetivos}

Analizar el resultado del proceso democrático de modificación del reglamento de ensayos clínicos pe- 
ruano, para determinar si en la actualidad se respeta la participación comunitaria y diversidad intercultural de nuestras poblaciones en la composición de los comités de ética.

\section{Resultados}

El propósito de introducir la ética en las discusiones y evaluación de proyectos de investigación es incorporar una visión sobre teorías morales disponibles y lineamientos éticos, cuidado y cautela de los derechos de los sujetos que participan y buenas prácticas clínicas. Perú no escapa a esta responsabilidad y para ello cuenta con una base normativa que sustenta la investigación.

\section{Crónica de un paso atrás en la regulación peruana luego de un proceso democrático de consulta:}

En Perú se establece que el desarrollo de las investigaciones se realice en el marco de las normas internacionales de ética en investigación; además, en el caso de la investigación clínica, existía una norma nacional específica que databa de 1982.
En 2006, el INS lanzó el Reglamento de Ensayos Clínicos, moderno, con buenas iniciativas que, sin embargo, tuvo observaciones provenientes de todos los actores involucrados en la investigación clínica. Por todo ello, el gobierno peruano inició un proceso de discusión, promoviendo la presentación de propuestas, correcciones, sugerencias, opiniones para la corrección y/o perfeccionamiento del reglamento de ensayos clínicos peruano $(3,6,7)$.

El Ministerio de Salud recibió diversas sugerencias o recomendaciones y, para un análisis más exhaustivo, conformó una comisión técnica. Ésta debía presentar al despacho ministerial un informe técnico en un plazo no mayor de 30 días contados desde su instalación.

En esta comisión no hubo representación nacional completa (no se consideraron representantes de provincias) pero por lo menos participaron otros representantes de la Red Nacional de Comité de Ética; Ministerio de Salud; Dirección General de Medicamentos, Insumos y Drogas; INS; Instituto Nacional de Ciencias Neurológicas; Foro Salud; Universidad Nacional Mayor de San Marcos y Academia Nacional de Medicina(4,5).

Tabla 1. Cronología del proceso de consulta pública para modificar el Reglamento de Ensayos Clínicos.

\begin{tabular}{|c|c|c|}
\hline Reglamento & Primer reglamento & Reglamento actual modificado \\
\hline Documento de aprobación & Decreto Supremo No 017-2006-S & Decreto Supremo No 006-2007-SA. \\
\hline Fecha de aprobación & 29 de julio de 2006 & 07 de junio de 2007 \\
\hline Proceso de consulta & $\begin{array}{l}\text { Se reciben discrepancias, observaciones y sugerencias } \\
\text { desde su aprobación. Por más de cinco meses } \\
\text { continuó observado. } \\
\text { Se presenta primer proyecto modificatorio. Y desde } \\
\text { el } 16 \text { de enero de } 2007 \text { se publica en el portal de } \\
\text { Internet del Ministerio de Salud por } 15 \text { días para } \\
\text { recibir las sugerencias o recomendaciones de la } \\
\text { opinión pública interesada. } \\
\text { El } 22 \text { de febrero de } 2007 \text { se reciben las observaciones } \\
\text { y sugerencias. }\end{array}$ & $\begin{array}{l}\text { El } 19 \text { de marzo de } 2007 \text { el Ministerio de Salud } \\
\text { crea una comisión técnica para analizar sugerencias } \\
\text { y observaciones al proyecto modificatorio del } \\
\text { Reglamento. } \\
\text { El } 13 \text { de abril de } 2007 \text { la comisión técnica eleva el } \\
\text { informe técnico final. } \\
\text { El } 07 \text { de junio de } 2007 \text { el Ministerio de Salud, } \\
\text { por decreto supremo, aprueba la modificación del } \\
\text { Reglamento. }\end{array}$ \\
\hline $\begin{array}{l}\text { Quienes participaron en la } \\
\text { creación del reglamento }\end{array}$ & $\begin{array}{l}\text { Red de Comités de Ética, Instituto Nacional de } \\
\text { Salud(INS), principalmente }\end{array}$ & $\begin{array}{l}\text { Comisión técnica de expertos, opinión pública en } \\
\text { general. Red de Comités de Ética, INS, otros. }\end{array}$ \\
\hline Artículos modificados & \multicolumn{2}{|c|}{$\begin{array}{l}\text { Aproximadamente } 33 \text { artículos y } 2 \text { disposiciones complementarias; se eliminaron } 2 \text { artículos y una } \\
\text { disposición complementaria y se agregaron además } 2 \text { disposiciones transitorias. Artículos modificados: } 7^{\circ} \text {, } \\
14^{\circ}, 19^{\circ}, 28^{\circ}, 29^{\circ}, 30^{\circ}, 32^{\circ}, 33^{\circ}, 34^{\circ}, 35^{\circ}, 39^{\circ}, 51^{\circ}, 52^{\circ}, 54^{\circ}, 56^{\circ}, 57^{\circ}, 58^{\circ}, 59^{\circ}, 60^{\circ}, 66^{\circ}, 67^{\circ}, 68^{\circ}, 69^{\circ} \text {, } \\
83^{\circ}, 89^{\circ}, 92^{\circ}, 93^{\circ}, 95^{\circ}, 104^{\circ}, 105^{\circ} \text { y el artículo } 131^{\circ} \text {. }\end{array}$} \\
\hline
\end{tabular}

Fuente: Elaboración propia y con apoyo bibliográfico extraído de los decretos supremos del Ministerio de Salud de Perú(6,7).

Desde que se aprobó la modificación del Reglamento de ensayos clínicos se ha iniciado una serie de acciones para implementar los cambios. Todo indica que la tarea es larga y que existen muchas cosas pendientes para hacer viable este reglamento. 
Regulación de la investigación clínica y comités de ética en Perú - Agueda Muñoz del Carpio Toia

Tabla 2. Resultados comparativos de las dos versiones del Reglamento de Ensayos Clínicos en la parte referida a la constitución de un Comité de Ética.

\begin{tabular}{|c|c|c|}
\hline Reglamento & Primer reglamento & Reglamento actual modificado \\
\hline Artículos modificados & $\begin{array}{l}\text { Constaba de } 137 \text { artículos y } 13 \text { disposiciones } \\
\text { complementarias. }\end{array}$ & $\begin{array}{l}\text { Consta de } 135 \text { artículos. Se modificaron } 33 \text { artículos y } 2 \\
\text { disposiciones complementarias. Se eliminaron } 2 \text { artículos y } \\
1 \text { disposición complementaria. Se agregaron } 2 \text { disposiciones } \\
\text { transitorias. }\end{array}$ \\
\hline Número & Mínimo 7 miembros. & Mínimo 5 miembros. \\
\hline $\begin{array}{l}\text { Constitución de un } \\
\text { comité de ética }\end{array}$ & $\begin{array}{l}\text { Los miembros podrán o no pertenecer a la } \\
\text { institución de investigación. } \\
\text { Al menos dos con formación en bioética. } \\
\text { Uno debe ser de la comunidad. } \\
\text { Al menos un abogado. }\end{array}$ & $\begin{array}{l}\text { Al menos un miembro de la institución. } \\
\text { Al menos un miembro que no pertenezca a la institución } \\
\text { y que no sea familiar inmediato de un miembro de la } \\
\text { institución de investigación. } \\
\text { Un miembro (1) de la comunidad. }\end{array}$ \\
\hline Suplentes & Igual número de suplentes. & Número de suplentes lo establecerá su reglamento interno. \\
\hline
\end{tabular}

Fuente: Elaboración propia y con apoyo bibliográfico extraído de los Decretos Supremos del Ministerio de Salud Perú $(6,7)$.

Como se puede observar, los requisitos para la participación de la comunidad en un comité de ética son más laxos en la regulación peruana vigente y no son discriminatorios. En la tabla 3 se presenta un análisis comparativo entre los principales documentos internacionales que tocan el tema de la constitución de un comité de ética.

Tabla 3. Resultados comparativos respecto de las recomendaciones internacionales y la regulación americana sobre la composición de comités de ética.

\begin{tabular}{|c|c|c|}
\hline Recomendaciones y regulaciones & Tamaño & Composición del comité \\
\hline $\begin{array}{l}\text { Guía } N^{\circ} 1 \text { de la UNESCO } \\
\text { publicada en } 2005 \text { referente a los } \\
\text { comités de ética. }\end{array}$ & $\begin{array}{l}\text { Variable, } \\
\text { de } 10 \text { a } 20 \\
\text { personas. }\end{array}$ & $\begin{array}{l}\text { Participación de profesionales de la salud, así como de no científicos (expertos en } \\
\text { bioética, abogados especializados en legislación médica, representantes del clero y } \\
\text { público de la comunidad local), todos ellos debidamente informados e interesados. }\end{array}$ \\
\hline $\begin{array}{l}\text { Guías Operacionales } \\
\text { para Comités de Ética en } \\
\text { Investigación Biomédica, OMS }\end{array}$ & $\begin{array}{l}\text { No especifica } \\
\text { número. }\end{array}$ & $\begin{array}{l}\text { Participación de miembros de diferente profesión. } \\
\text { Ambos sexos. } \\
\text { Al menos un miembro cuya área primaria de experiencia sea un área no científica. } \\
\text { Al menos un miembro independiente de la institución en donde se realice la } \\
\text { investigación. } \\
\text { Deben ser multidisciplinarios y multisectoriales en su composición, e incluir expertos } \\
\text { científicos relevantes, balanceados en edad y sexo, y personas que representen los } \\
\text { intereses y preocupaciones de la comunidad. Deben establecerse conforme a los } \\
\text { reglamentos y leyes del país y, además, de acuerdo con los valores y principios de la } \\
\text { comunidad a la que sirven. }\end{array}$ \\
\hline $\begin{array}{l}\text { ICH-GCPs } \\
\text { Lineamientos para la Buena } \\
\text { Práctica Clínica (BPC) }\end{array}$ & $\begin{array}{l}\text { Se recomienda } \\
\text { por lo } \\
\text { menos cinco } \\
\text { miembros. }\end{array}$ & $\begin{array}{l}\text { Considerar al menos un miembro cuya área primaria de interés sea no científica; } \\
\text { asimismo, al menos un miembro independiente de la institución/sitio donde se } \\
\text { llevará a cabo el estudio. }\end{array}$ \\
\hline $\begin{array}{l}\text { FDA 21CFR Part } 56 \text { Sub part } \\
\text { B 56.107 Institutional Review } \\
\text { Board (IRB) Membership }\end{array}$ & 5 miembros. & $\begin{array}{l}\text { Que sean comités con representación de género, raza, bagaje cultural y sensibilidad } \\
\text { para entender las actitudes de la comunidad. Debe incluir personas con conocimiento } \\
\text { en diferentes áreas. Al menos un miembro del área científica, uno no científico, por } \\
\text { lo menos que no pertenezca a la institución y que no sea familiar inmediato de una } \\
\text { persona afiliada a la institución. }\end{array}$ \\
\hline $\begin{array}{l}\text { CFR OHRP Title } 45 \text { Part } 46 . \\
107 \text { Membresía en un IRB }\end{array}$ & 5 miembros. & $\begin{array}{l}\text { Cuando menos cinco miembros con experiencias diversas para promover una revisión } \\
\text { completa y adecuada de las actividades de la investigación comúnmente conducidas por } \\
\text { la institución. Estará suficientemente calificado mediante la experiencia, competencia } \\
\text { y diversidad de sus miembros, incluyendo raza, género, antecedentes culturales y } \\
\text { sensibilidad respecto de cuestiones como actitudes de la comunidad. }\end{array}$ \\
\hline
\end{tabular}

Fuente: Elaboración propia con apoyo bibliográfico(8-10). 
Como se puede observar en las guías, recomendaciones y regulaciones internacionales respetan valores y principios de la comunidad, sin exigir requisitos que podrían discriminar o sesgar su participación.

Los cambios más notorios que ha sufrido la regulación peruana en 2007 se registran en la estructura de los comités de ética (IRB); los principales son:

- En la regulación 2006 se requería de mínimo de siete miembros y entre ellos un abogado; actualmente sólo se requiere cinco miembros y no se requiere de un abogado.

- La capacitación de los miembros debe ser actualmente sobre aspectos científicos, éticos y legales de la investigación; la anterior versión indicaba además aspectos farmacológicos.

- Respecto de la inclusión de miembros de la comunidad dentro de los comités de ética, también se observan grandes cambios, ya que ahora el requisito para que una persona sea elegida como representante es que no pertenezca a la institución de investigación y no tenga una profesión en ciencias de la salud. En la versión anterior de la regulación se exigía que cuando menos un miembro debía ser de la comunidad, que no perteneciera al campo de la salud ni a la institución; pero el requisito mínimo para su incorporación era que debía tener educación secundaria completa y a propuesta de una organización social de servicio que realizara acciones en favor del desarrollo de la comunidad. Este requisito era claramente diferente a la estructura que recomienda la ICH-GCPs, las Guías CIOMS y Guía UNESCO 2005 para comités de ética.

- Otra de las modificaciones en este nuevo reglamento se refiere a la posibilidad que tienen ahora los comités de ética de cobrar una tarifa destinada a cubrir gastos operativos (Artículo 57 ${ }^{\circ}$.

- El reglamento peruano sugiere también que cada institución de investigación puede constituir un comité de ética de investigación y registrarlo en el INS. También en este documento se abre la posibilidad de que un investigador pueda hacer uso de otros comités de ética registrados en el INS que acepten cumplir con tal fin.

\section{Discusión}

La principal función de un comité de ética en investigación es proteger los derechos, el bienestar y la seguridad de los participantes, garantizando que los métodos utilizados no los expongan a riesgos innecesarios. En su constitución debe estar representada la sociedad, la comunidad de enfermos, entre otros, ya que son ellos los principales actores sociales en la ejecución de la investigación y pueden apoyar a la vigilancia ética para evitar explotación. La participación de la comunidad es también importante porque su presencia podría fortalecer los esfuerzos al momento de negociar los beneficios justos en la investigación. Ruth Macklin afirma que una forma de explotación se da cuando las personas o los patrocinadores se aprovechan de la pobreza, debilidad o dependencia de los otros, usándolos para servir a sus propias metas, sin beneficios adecuados para compensar a los individuos o a los grupos que son dependientes o menos poderosos(11). Luego de la modificación del Reglamento de Ensayos Clínicos se ha logrado la inclusión de representantes de la comunidad en los comités de ética.

En el Reglamento del 29 de julio de 2006 algunos de los artículos creaban un ambiente de inequidad y exclusión de ciertos grupos para participar en comités de ética. Según esa regulación, el comité institucional de ética en investigación se constituye teniendo en cuenta lo siguiente: un (1) miembro cuando menos debe ser de la comunidad, que no pertenezca al campo de la salud, ni a la institución. Deben ser requisitos mínimos para su incorporación: educación secundaria completa y a propuesta de una organización social de servicio, sin fines de lucro y que realiza acciones a favor del desarrollo de la comunidad(4).

Este era un prerrequisito discriminatorio para un gran porcentaje de la población peruana y sobre todo para las poblaciones nativas, selváticas, de la sierra y de áreas rurales. Muchos peruanos no tienen el nivel secundario completo por varias razones: falta de acceso a la educación, ingreso temprano al mundo laboral, decisión personal de no concluir la secundaria por otras alternativas, etc. Era discriminatorio ya que sabemos que nivel de escolaridad no es lo mismo que acervo cultural. Perú es un país pluriétnico y pluricultural, y con gran acervo. Esto debe respetarse al momento de incluir a un representante de la comunidad en la estructura de un comité de ética independiente que evaluará los riesgos y beneficios de una investigación en un grupo de esa comunidad, o la aplicabilidad y nivel de entendimiento de un consentimiento informado, entre otros aspectos.

La constitución del comité institucional de ética de la investigación debe estar balanceada en términos genera- 
cionales, de edad y de género, para tratar de representar de la mejor manera los intereses y preocupaciones de la comunidad. Debemos recordar que en Perú existen más de tres millones de peruanos iletrados. De acuerdo con información del Ministerio de Educación, en 2006, del total de la población iletrada, $64 \%$ eran mujeres y $54 \%$ vive en las áreas rurales. Se evidencia que el analfabetismo se concentra en las seis principales regiones del país, incluidas áreas andinas y selváticas, las cuales concentran a casi 1 millón 500 mil personas. ¿Esta población era una cifra menor y por eso debía ser excluida?

En cuanto a un análisis ético, se podría hablar de un doble estándar. El argumento del primer reglamento era: no es conveniente que un miembro representante de la comunidad ante un comité de ética sea una persona que no haya concluido sus estudios secundarios, porque no puede entender todo el proceso en el que se revisa un protocolo. Sin embargo, estas poblaciones son las que, en gran número, participan en una investigación científica; para esta circunstancia sí se les calificó como aptos.

Luego de la consulta al reglamento y su posterior modificación, el requisito discriminatorio fue eliminado, por lo que, a partir del 7 de junio de 2007, cualquier persona que pueda representar a la comunidad puede hacerlo, independientemente de su nivel educativo.

\section{Conclusiones}

Se puede señalar que Perú cuenta ahora con un Reglamento de Ensayos Clínicos con latos estándares de protección para los participantes de una investigación clínica.

Perú está preparado para el reto de la globalización de la investigación, es decir, ha sentado las bases éticas, administrativas y científicas para la realización de investigaciones con patrocinadores locales e internacionales, y que guardan relación con las regulaciones internacionales.

Ha quedado un aprendizaje democrático luego del proceso de modificación del reglamento en la sección referida a participación de la comunidad en los comités de ética, y este logro es saludable para todos los peruanos, para el destino de la investigación, para la cooperación internacional en investigación y para la inversión de laboratorios internacionales: resultado de la participación abierta de la sociedad.

Es importante crear espacios de consulta, de diálogo, de sana discrepancia y de propuesta. También, poner a disposición reglamentos claros, viables, factibles, actuales, justos para todos los involucrados y que no posterguen o discriminen a la comunidad y su diversidad intercultural.

Deseo expresar mi agradecimiento especial a WIRB, a WHO y a la fundación Middelton de estudios éticos de Estados Unidos por el apoyo recibido durante la beca de entrenamiento en ética de la investigación y protección de los seres humanos. De manera especial, al Dr. Humberto Guerra y a Milena Toia-Larsen.

\section{Referencias}

1. Emanuel E, Wendler D, Grady C. What makes clinical research ethical? JAMA 2000; 283: 2701-2711.

2. Gracia D. (1998). Investigación Clínica, conceptos fundamentales. En Gracia D. Ética y vida. Bogotá: El Búho; 1998: $77-110$.

3. UNESCO. Guía No 1: Establishing Bioethics Committees. UNESCO; 2005.

4. Reglamento de Ensayos Clínicos Perú. Decreto supremo 017-2006-SA -29 de julio del 2006. Disponible en: http://www. ins.gob.pe/gxpsites/agxppdwn.aspx?2,13,331,O,S,0,1257;S;1;137, REGLAMENTO DE ENSAYOS CLÍNICOS EN EL PERÚ aprobado con D.S. No 017-2006-SA.

5. Reglamento de Ensayos Clinicos Modificado Perú. Decreto supremo 006-2007-SA -08 de junio del 2007. Disponible en: http://www.ins.gob.pe/gxpsites/agxppdwn.aspx?2,13,64,O,S,0,1846;S;1;137,

6. Lurie P, Wolfe SM. Unethical trials of interventions to reduce perinatal transmission of the human immunodeficiency virus in developing countries. N Engl J Med 1997; 337: 801-808.

7. WHO/TDR Guias operacionales para comités de ética que evaluan investigación biomédica. Ginebra: Organización Mundial de la Salud; 2000.

8. Council for International Organizations of Medical Sciences. International Ethical Guidelines for Biomedical Research Involving Human Subjects. Ginebra: CIOMS; 2000. 
9. Conferencia Internacional de Armonización. Guía tripartita armonizada de la Conferencia Internacional de Armonización. Lineamientos para la buena práctica clinica (BPC) sobre requerimientos técnicos para el registro de productos farmacéuticos para uso en humanos. Conferencia Internacional de Armonización (CIARM (C ICH); 1997. Disponible en: http://www. fda.gov/cder/guidance/959fnl-spanish.pdf

10. Krugman S, et al. Infectious Hepatitis. JAMA 1967; 200(5): 365-373.

11. Ruth Macklin. Ética de la investigación internacional: el problema de la justicia hacia los países menos desarrollados. Acta Bioethica 2004; 10(1): 27-37.

Recibido: 11 de abril de 2008

Aceptado: 22 de junio de 2008 